\title{
Cauterização química de granulomas periestomais com ácido tricloroacético a 50\%
}

\author{
Chemical cauterization of peristomal granulomas with 50\% trichloroacetic acid
}

Cauterización química de granulomas periestomales con ácido tricloroacético a 50\%

Rosaura Soares Paczek ${ }^{1 *}$, Luisa Zadra Passberg²

ORCID IDs

Paczek RS (D) https://orcid.org/0000-0002-4397-1814

Passberg LZ (D) https://orcid.org/0000-0002-8934-834X

\section{COMO CITAR}

Paczek RS; Passberg LZ. Cauterização química de granulomas periestomais com ácido tricloroacético a 50\%. ESTIMA, Braz. J. Enterostomal Ther., 17: e0319. https://doi.org/10.30886/estima. v17.641 PT

\begin{abstract}
RESUMO
Objetivo: Este estudo aborda o tratamento dos granulomas, uma das possíveis complicações das estomias, os quais são caracterizados como lesões focais, cuja formação ocorre devido à presença de agentes agressores aos tecidos a partir do aumento do grau de celularidade e de outros elementos teciduais, gerando incômodo e ansiedade aos pacientes. Tal estudo tem como objetivo descrever os resultados clínicos do tratamento de granulomas periestomais com o uso de ácido tricloroacético (ATA) a 50\%. Métodos: Trata-se de estudo de série de casos, vivenciado por profissionais em um centro de referência para tratamento de indivíduos com estomia no município de Porto Alegre, estado do Rio Grande do Sul. Resultados: A amostra do estudo foi constituída de 13 pacientes que realizaram acompanhamento para tratamento de granulomas periestomais com o ATA. A coleta de dados foi realizada durante consultas de enfermagem, a partir da observação e registro fotográfico dos granulomas, com posterior evolução no prontuário individual. Conclusão: Concluiu-se, a partir deste estudo, que o tratamento regular com o ATA resultou em regressão dos granulomas até seu total desaparecimento, podendo tal ácido ser considerado, assim, potencial opção terapêutica; porém, recomenda-se a realização de mais estudos sobre sua utilização na estomaterapia, buscando a utilização de metodologias de pesquisa clínica mais robustas e com controle de vieses.
\end{abstract}

DESCRITORES: Granuloma; Cauterização; Estomia; Estomaterapia. 


\begin{abstract}
Objetive: This study deals with the treatment of granulomas, one of the possible complications of the stomies, which are characterized as focal lesions, whose formation occurs due to the presence of aggressive agents to the tissues from the increase of the degree of cellularity and other tissue elements, generating annoyance and anxiety to patients. This study aims to describe the clinical results of the treatment of peristomal granulomas with the use of 50\% trichloroacetic acid (TCA). Methods: This is a case series study, carried out by professionals in a referral center for the treatment of individuals with an ostomy in the city of Porto Alegre, state of Rio Grande do Sul. Results: The study sample consisted of 13 patients who underwent follow-up for treatment of peristomal granulomas with TCA. Data collection was performed during nursing consultations, based on the observation and photographic record of the granulomas, with subsequent evolution in the individual charts. Conclusion: It was concluded from this study that the regular treatment with TCA resulted in regression of the granulomas until their total disappearance, being this acid able to be considered, therefore, a potential therapeutic option; however, it is recommended to carry out further studies on its use in stomatherapy, seeking the use of more robust clinical research methodologies and with bias control.
\end{abstract}

DESCRIPTORS: Granuloma; Cauterization; Ostomy; Stomatherapy.

\title{
RESUMEN
}

Objetivo: Este estudio aborda el tratamiento de los granulomas, una de las posibles complicaciones de las estomias, que se caracterizan como lesiones focales, cuya formación ocurre debido a la presencia de agentes agresores a los tejidos a partir del aumento del grado de celularidad y de otros elementos teciduales, generando incomodidad y ansiedad a los pacientes. Este estudio tiene como objetivo describir los resultados clínicos del tratamiento de granulomas periestomales con el uso de ácido tricloroacético (ATA) al 50\%. Métodos: Se trata de estudio de serie de casos, vivido por profesionales en un centro de referencia para el tratamiento de individuos con estomia en el municipio de Porto Alegre, estado de Rio Grande do Sul. Resultados: La muestra del estudio fue constituida de 13 pacientes que realizaron seguimiento para tratamiento de granulomas periestomales con el ATA. La recolección de datos fue realizada durante consultas de enfermería, a partir de la observación y registro fotográfico de los granulomas, con posterior evolución en el prontuario individual. Conclusión: Se concluyó, a partir de este estudio, que el tratamiento regular con el ATA resultó en regresión de los granulomas hasta su total desaparición, pudiendo tal ácido ser considerado, así, potencial opción terapéutica; sin embargo, se recomienda realizar más estudios sobre su utilización en la estomaterapia, buscando la utilización de metodologías de investigación clínica más robustas y con control de sesgos.

DESCRIPTORES: Granuloma; cauterización; ostomía; Estomaterapia..

\section{INTRODUÇÃO}

Os granulomas são caracterizados como lesões focais, cuja formação ocorre devido à presença de agentes agressores aos tecidos, a partir do aumento do grau de celularidade e de outros elementos teciduais ${ }^{1}$. Acredita-se que, em relação aos granulomas periestomais, a reação ocorrida seja desencadeada pela irritação da pele nos locais de sutura, ou ainda pela retenção dos fios de sutura por tempo prolongado ${ }^{2-4}$. Além disso, granulomas periestomais podem surgir como resultado de irritação cutânea originada a partir do contato com efluentes, como fezes e urina ${ }^{5}$.

Observa-se a presença de granulomas em pacientes com estomia tanto no pós-operatório mediato quanto no tardio, sendo que esses podem surgir em pontos isolados, múltiplos ou ao redor de toda a estomia ${ }^{3}$. Tais tecidos são ricos em capilares em sua superfície, o que faz com que sangrem com facilidade ao serem manuseados ${ }^{1}$. Devido às suas características macroscópicas, permitem o diagnóstico visual pelo profissional da saúde, porém, também podem acabar tornando-se geradores de incômodo e ansiedade por parte dos pacientes 6 .

Tradicionalmente, os granulomas periestomais têm sido tratados a partir do uso de nitrato de prata, produto que se tornou a primeira escolha de tratamento, por favorecer rápida aplicação e ser indolor ao paciente ${ }^{3-6}$. Caso o tratamento com nitrato de prata não seja efetivo, opta-se pelo uso de corticosteroides tópicos e/ou de nitrogênio líquido (crioterapia). O ácido tricloroacético (ATA), por sua vez, comumente utilizado em especialidades como dermatologia, odontologia, ginecologia e urologia para tratamento de unhas encravadas, lesões condilomatosas orais, vaginais e anais, realização de peeling químico, entre outras, pode também ser aplicado na estomaterapia com objetivo de remover granulomas, a partir da cauterização química $^{7-12}$. Tal ácido, comumente utilizado na forma de 
solução, também pode ser encontrado na apresentação de pasta ou gel ${ }^{13}$.

Segundo a Sociedade Brasileira de Dermatologia, o tratamento com ATA consiste na aplicação do ácido sobre determinados tipos de lesões, com objetivo de regressão e posterior remoção $0^{12}$. Apesar de bastante utilizado, há grande dificuldade de encontrar referências que abordem o uso do ATA, assim como os próprios granulomas periestomais. Autoras explicam que os motivos para tão poucas publicações podem estar relacionados ao fato de que os granulomas não necessariamente causam desconforto físico aos pacientes, geralmente tratando-se de lesões indolores, resultando em pouco interesse em pesquisas os envolvendo, além de suas modalidades terapêuticas ${ }^{3}$. Nesse sentido, tal estudo torna-se fundamental, uma vez que trará informação e servirá de base científica para conhecimento e tratamento dos granulomas.

Para o tratamento de granulomas periestomais, propõe-se o tratamento com o ATA na concentração de $50 \%$, com objetivo de regressão completa das lesões sem danos aos pacientes. Tradicionalmente, o ATA é utilizado em concentrações que variam de 10 a 70\%, porcentagem essa que influencia em sua penetração na lesão; porém, sabe-se que o tratamento com concentrações superiores a $50 \%$ está relacionado ao maior número de cicatrizes e alterações na coloração da pele no local de aplicação ${ }^{13}$. O processo de cauterização química de lesões com esse ácido costuma ser realizado uma vez por semana até completa resolução, e recomenda-se que seja efetuado por profissional capacitado e mediante o estabelecimento de protocolo institucional, conforme estabelecido pelo Conselho Federal de Enfermagem de São Paulo para tratamento de lesões condilomatosas $^{14,15}$.

Devido à sua ação cáustica, que resulta em morte celular por desnaturação proteica, o ATA faz com que as lesões se tornem esbranquiçadas logo após sua aplicação $0^{13}$. A irritação local provocada pelo uso do ácido faz com que alguns pacientes sintam ardência ou dor durante o procedimento, e podem ocorrer casos de necrose do tecido adjacente, assim como de ulceração9. Nesse sentido, é necessário ressaltar que a pele periestomal e também a mucosa da estomia devem ser preservadas do ácido, e por isso a aplicação por profissional habilitado e de forma cautelosa é indispensável. Durante a aplicação do ATA, a preservação dos tecidos adjacentes geralmente é feita com o uso de vaselina ou de materiais protetores, como os compostos de hidrocoloide.
Autores recomendam, também, a disponibilidade de agentes neutralizantes, como o bicarbonato de sódio, para os casos de excessos de aplicação ${ }^{9}$.

\section{OBJETIVOS}

Descrever os resultados clínicos do tratamento de granulomas periestomais em pacientes com estomia com o uso de ATA a $50 \%$.

\section{MÉTODOS}

Trata-se de estudo de série de casos vivenciado por profissionais em um centro de referência para tratamento de indivíduos com estomia no município de Porto Alegre, estado do Rio Grande do Sul, realizado no período de abril a junho de 2017. Em 2013, ano em que foi realizada a investigação de dados do serviço, esse tinha, em média, 791 prontuários ativos, sendo 396 (50,06\%) usuários do sexo masculino e 395 (49,9\%) do sexo feminino. A maior parte deles [512 (64,72\%)] tinha idade igual ou superior a 60 anos, enquanto $259(32,74 \%)$ enquadravam-se na faixa etária de 19-59 anos, cinco (0,63\%) entre 12-18 anos e 17 $(2,14 \%)$ entre $0-12$ anos. Quanto ao tipo de estomia, as colostomias eram as mais frequentes, sendo apresentadas por $463(58,53 \%)$ usuários, seguidas das ileostomias [151 $(19,08 \%)]$, urostomias [68 (8,59\%)], nefrostomias [4 (0,5\%)] e fístulas $[2(0,25 \%)]$.

Para coleta de dados, seguiram-se os critérios de inclusão: pacientes cadastrados no serviço do estudo que estivessem em acompanhamento em consulta de enfermagem para o tratamento de granulomas periestomais. Foram critérios de exclusão: pacientes cadastrados em situação de desistência ou abandono do serviço; indivíduos sem granulomas periestomais; e os que se recusaram a participar do estudo. Os granulomas, a partir de suas características peculiares, eram identificados e diagnosticados visualmente durante as consultas com o enfermeiro estomaterapeuta. Sendo assim, a amostra deste estudo é de 13 pacientes que preencheram plenamente os critérios de inclusão estabelecidos.

A coleta de dados foi realizada durante as consultas de enfermagem, a partir da observação e do registro fotográfico dos granulomas, com posterior evolução no prontuário individual. Nesse sentido, houve acompanhamento dos 
pacientes pelo enfermeiro estomaterapeuta, verificando se houve ou não regressão do granuloma pelo uso do ATA. Nos casos de pacientes faltosos às consultas agendadas, fez-se contato telefônico, com objetivo de alertá-los sobre a periodicidade das consultas e a importância do tratamento regular. Após coleta, os dados foram organizados em planilha, analisados e apresentados por meio de estatística descritiva.

Importante destacar que o ATA a 50\% foi adquirido em farmácia de manipulação e a escolha pela sua utilização foi motivada por sua praticidade, efetividade descrita na literatura e baixo custo, além de dificuldades no acesso a produtos como o nitrato de prata no mercado. Para sua cuidadosa aplicação, utilizou-se cotonete embebido na solução.

O estudo foi aprovado pelo Comitê de Ética em Pesquisa da Secretaria Municipal de Saúde da Prefeitura Municipal de Porto Alegre sob parecer no 2.091.735. No desenvolvimento deste projeto, respeitaram-se os devidos aspectos éticos, observando-se rigorosamente a Resolução 466/12 do Conselho Nacional de Saúde, que dispõe sobre as diretrizes e normas regulamentadoras de pesquisas envolvendo seres humanos ${ }^{16}$.

É relevante ressaltar que os dados somente foram obtidos após o esclarecimento aos participantes dos objetivos da pesquisa e dos seus direitos, por meio do Termo de Consentimento Livre e Esclarecido, assinado por eles e pelas pesquisadoras envolvidas. Além disso, para autorização dos registros fotográficos e uso de imagens, os pacientes preencheram um termo específico.

\section{RESULTADOS}

Foram atendidos 13 pacientes com granulomas periestomais, os quais aceitaram participar do estudo. Quanto à caracterização dos indivíduos, houve predomínio do sexo feminino, sendo nove mulheres $(69,2 \%)$, e as idades dos participantes variaram entre 4 e 84 anos, com idade média de 59,54 anos. Prevaleceu a colostomia dentre os tipos de estomia, presente em oito (61,5\%) indivíduos. Com relação às doenças-base para confecção da estomia, predominaram as neoplasias, apresentadas por nove $(69,2 \%)$ indivíduos, sendo identificadas também doença diverticular, paralisia cerebral e hidronefrose. A única paciente pediátrica participante do estudo, com 4 anos de idade, apresentava gastrostomia devido à paralisia cerebral.

$\mathrm{O}$ número de aplicações do ATA variou entre uma a oito. Oito $(61,5 \%)$ pacientes fizeram tratamento regular com o ATA semanalmente, até a completa regressão das lesões granulomatosas, enquanto cinco $(38,5 \%)$ iniciaram o tratamento, porém não retornaram às consultas para avaliar a necessidade de completá-lo (Tabela 1).

Tabela 1. Relação entre o número de participantes e o número de aplicações do ácido tricloroacético (ATA) para resolução do granuloma.

\begin{tabular}{ccc} 
Aplicações do ATA & \multicolumn{2}{c}{ Participantes } \\
\cline { 2 - 3 }$(\mathrm{n})$ & $(\mathrm{n})$ & $(\%)$ \\
\hline 2 & 3 & 37,5 \\
\hline 2 & 1 & 12,5 \\
\hline 5 & 3 & 37,5 \\
\hline 8 & 1 & 12,5 \\
\hline
\end{tabular}

Dentre os cinco indivíduos que não retornaram ao serviço para nova avaliação, três (60\%) aplicaram o ATA apenas uma vez, enquanto os outros dois (40\%) aplicaram três vezes, sem registro de resolução da lesão. Não foram registradas complicações a partir do uso do ATA no presente estudo.

A Fig. 1 apresenta alguns dos registros fotográficos realizados durante as consultas dos pacientes atendidos, feitos com objetivo de compará-los semanalmente para avaliar a eficácia do ATA na regressão e resolução dos granulomas periestomais.

Figura 1. Imagens fotográficas feitas para avaliar a regressão dos granulomas periestomais durante o tratamento com o ácido tricloroacético (ATA).

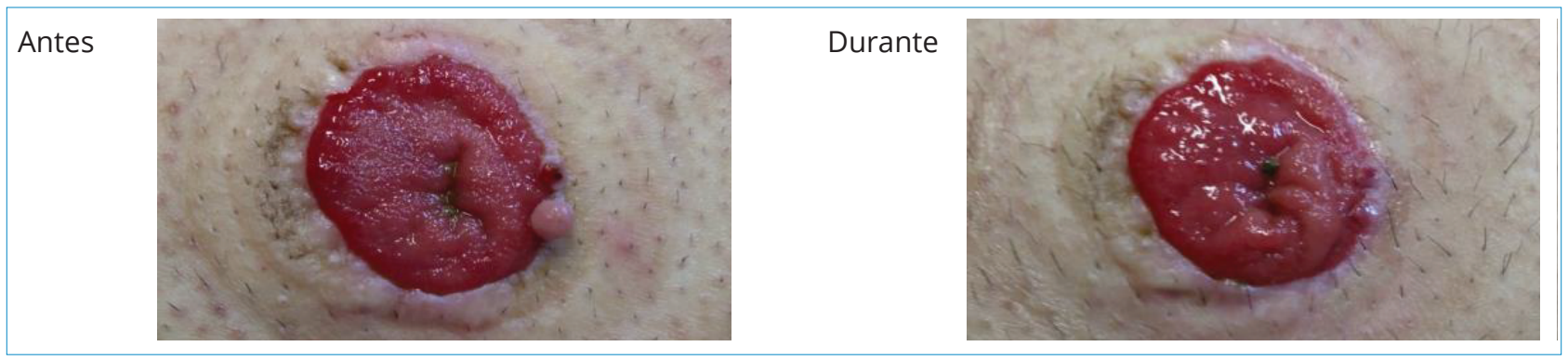


Figura 1. Continuação...

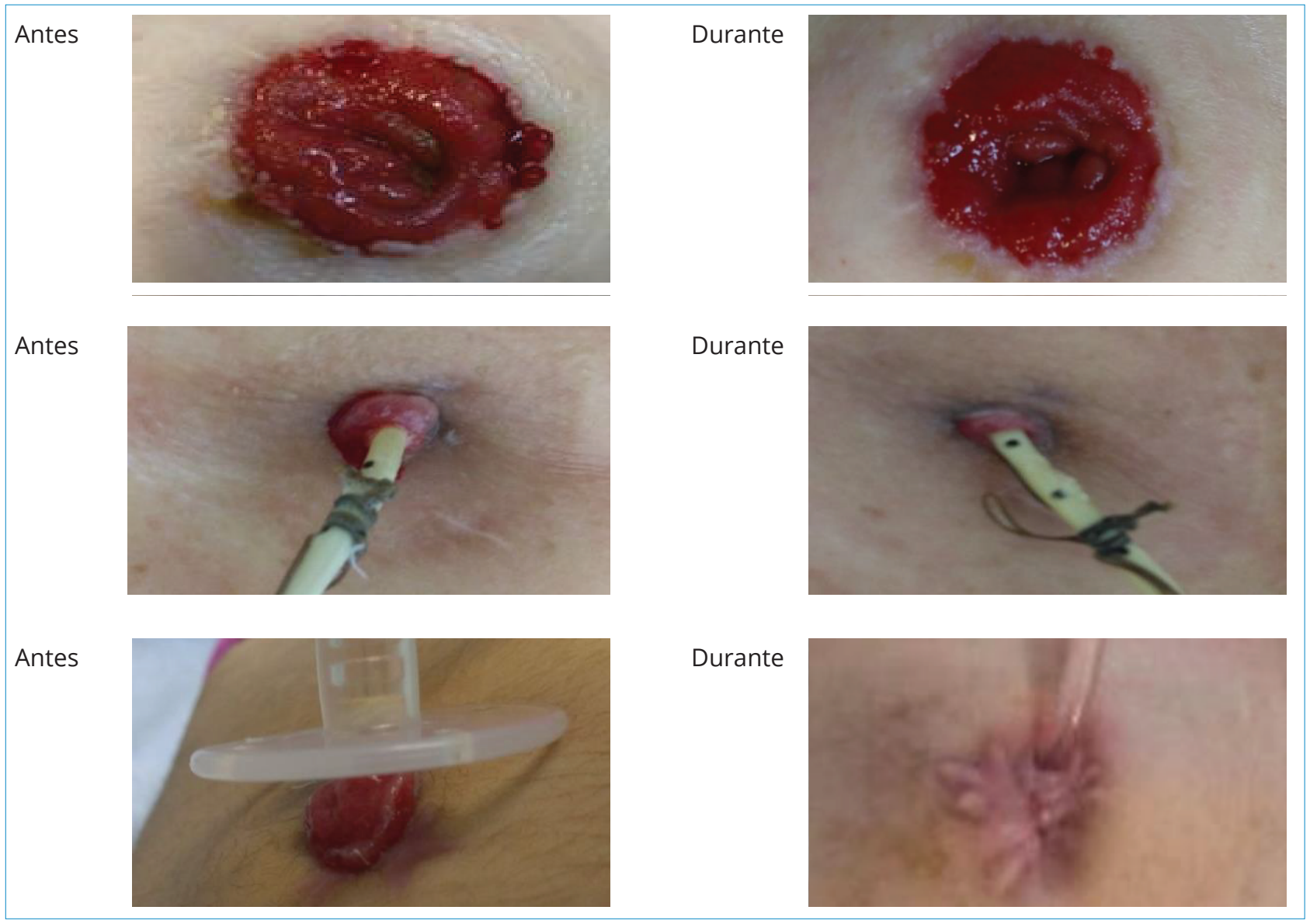

Fonte: arquivo pessoal das autoras.

\section{DISCUSSÃO}

Pesquisa realizada no estado de Minas Gerais em 2010 apontou a predominância do sexo feminino $(66,7 \%)$ entre os indivíduos com estomia ${ }^{17}$, o mesmo ocorrendo em Teresina, estado do Piauí, onde estudo encontrou $62,50 \%$ de mulheres em sua amostra ${ }^{18}$, dados esses que vão ao encontro ao identificado entre os indivíduos do presente estudo. Quanto à faixa etária, estudo aponta a idade média de 61,6 anos entre a população com estomia estudada ${ }^{17}$, pouco mais do que os 59 anos encontrados. Segundo Boccardo et al. ${ }^{19}$, há predomínio de indivíduos com estomia na faixa etária entre 58 e 78 anos e, conforme Macedo et al. ${ }^{18}$, isso pode ser explicado pelo fato de que a idade superior a 40 anos configura-se como fator de risco para o aparecimento de doenças como o câncer, que por vezes tem, como parte do tratamento, a confecção da estomia.

Com relação à caracterização da estomia, estudo identificou predominância de pacientes com colostomia
$(81,25 \%)$, seguidos de urostomias $(12,50 \%)$ e de ileostomias $(6,25 \%)^{18}$. Sabe-se que a realização da estomia está relacionada diretamente ao tratamento de uma comorbidade que pode acometer diferentes órgãos e sistemas corporais ${ }^{20}$. Nesse sentido, tais resultados encontrados diferem em parte dos identificados no presente estudo, em que as patologias do sistema urinário foram inferiores às intestinais em geral. Porém, é concordante o fato de que a maior parte das estomias está localizada no cólon, ou seja, no intestino grosso.

Também em outros estudos, a neoplasia foi considerada a doença-base mais prevalente para confecção da estomia, seguida por doenças inflamatórias ${ }^{17,18}$. O câncer é considerado importante problema de saúde pública, uma vez que, a cada ano, são diagnosticados milhões de novos casos no mundo ${ }^{21}$. No Brasil, o número estimado em 2014 e 2015 foi de aproximadamente $576 \mathrm{mil} \mathrm{casos}^{22}$. Sabe-se que, assim como em outras doenças, o desenvolvimento dos cânceres resulta de uma complexa interação entre fatores genéticos e ambientais. Com relação às neoplasias de cólon e reto, 
para as quais a colostomia pode ser indicada como parte do tratamento, a cada ano são diagnosticados cerca de um milhão de novos casos mundialmente ${ }^{21}$.

Observa-se, ao longo das duas últimas décadas, o aumento do número de casos de paralisia cerebral na infância, o que pode estar relacionado à melhoria nos cuidados perinatais, que aumentam a sobrevivência de crianças prematuras e de baixo peso extremo. Além de causar deficiências neuromotoras, a paralisia cerebral pode resultar em prejuízos ao desempenho de atividades cotidianas, como se alimentar sozinho $^{23}$. A gastrostomia, nesse sentido, tem como uma das indicações mais comuns os problemas de causa neurológica e é utilizada como meio de substituição à sonda nasoentérica em pacientes que necessitam receber aporte nutricional por tempo prolongado ${ }^{24}$, como a participante desse estudo.

Com relação ao tratamento dos granulomas periestomais com ATA, observou-se, no presente estudo, que a necessidade de aplicações estava relacionada ao tamanho e à quantidade de granulomas em torno do estomia. Nesse sentido, indivíduos que apresentavam granulomas maiores ou em maior quantidade necessitaram de maior número de aplicações para a resolução, enquanto granulomas menores eram resolvidos com mais facilidade a partir de menor número de aplicações do ATA. Além disso, nos casos em que os agentes agressores entravam novamente em contato com os tecidos após o procedimento, como em situações de extravasamento de efluentes e de fricção de tubos/cateteres com a pele periestomal, foram necessárias mais aplicações do ácido, visando à resolução da lesão. Como os granulomas não costumam causar grande incômodo e dor aos indivíduos, suspeita-se que a baixa adesão ao tratamento regular nesse estudo esteja relacionada a esse fato.

O tratamento de lesões com o uso do ATA é uma opção terapêutica favorável, pouco traumática aos pacientes e de custo acessível, porém que precisa ser mais estudada, tendo em vista que atualmente existem poucas referências, principalmente na área da estomaterapia. Estudos que utilizaram o ATA para tratamento de lesões condilomatosas apontam que tal ácido é mais eficaz para o tratamento de lesões de pequenas dimensões, e que tem, nesse sentido, taxa de resposta de até $80 \%$, obtendo, assim, bons resultados de cura. Outro ponto positivo destacado por autores é o fato de que o ATA não é absorvido sistemicamente ${ }^{9}$.

Sendo assim, pacientes que realizaram tratamento para granulomas periestomais regularmente com o uso do ATA obtiveram regressão das lesões até seu total desaparecimento. Há de se considerar, porém, que o uso do ATA apresenta alguns riscos, assim como ocorre com outros materiais compostos por ácidos, podendo causar queimaduras acidentais, o que pode ser minimizado com o uso da técnica e materiais adequados ao procedimento, além de recursos humanos capacitados.

\section{CONCLUSÃO}

A partir deste estudo, observou-se que o tratamento regular com ATA teve efeito positivo para a regressão dos granulomas até seu total desaparecimento. Tal resultado pode favorecer o cuidado aos pacientes com estomia, tendo em vista que o ATA consiste em uma alternativa eficaz de tratamento, com valor acessível e de fácil aplicação. Recomenda-se, portanto, a realização de mais estudos sobre a utilização do ATA na estomaterapia, buscando a utilização de metodologias de pesquisa clínica mais robustas e com controle de vieses.

\section{CONTRIBUIÇÃO DOS AUTORES}

\author{
Conceitualização, Paczek RS e Passberg LZ; Metodologia,
} Paczek RS e Passberg LZ; Investigação, Paczek RS e Passberg LZ; Redação - Primeira versão, Paczek RS e Passberg LZ; Redação - Revisão \& Edição, Paczek RS e Passberg LZ; Recursos, Paczek RS; Supervisão, Paczek RS.

\section{REFERÊNCIAS}

1. Corrêa L. Inflamação crônica e granulomas. São Paulo: Universidade de São Paulo; 2000

2. Zulkowski K. Ostomy terms and definitions. WCET. 2015;35(3):48-50.
3. Yamada BFA, Peres CSV, Santos VLCG. Granuloma em gastrostomia: uma alternativa inovadora de tratamento tópico. ESTIMA, Braz J Enterostomal Ther. 2004;2(4):11-4.

4. Steinhagen E, Colwell J, Cannon LM. Intestinal stomas postoperative stoma care and peristomal skin complications. 
Clin Colon Rectal Surg. 2017;30(3):184-92. https://doi. org/10.1055/s-0037-1598159

5. Monteagudo B. Hipergranulación periestomal. Med Cutan Iber Lat Am. 2009;37(1):64-6.

6. Dukes S, Lowther C, Martin T, Osborne D. Guidelines for treating stoma granulomas at the mucocutaneous junction. Gastroenterol Nurs. 2010:8(1):16-21. https://doi. org/10.12968/gasn.2010.8.1.46674

7. Ricardo HJ, Lorduy MC, Caballero AD. Efectividad de la terapia con ácido tricloroacético en el tratamiento de lesiones en la hiperplasia epitelial focal. Rev Odont Mex. 2016;20(4): 23076. https://doi.org/10.1016/j.rodmex.2016.11.004

8. Barreiros H, Matos D, Goulão J, Serrano P, João A, Brandão FM. Using $80 \%$ trichloroacetic acid in the treatment of ingrown toenails. An Bras Dermatol. 2013;88(6):889-93. https://doi.org/10.1590/abd1806-4841.20132296.

9. Brás F, Sardinha R, Pacheco A. Modalidades terapêuticas no tratamento dos condilomas acuminados. Acta Obstet Ginecol Port. 2015;9(5):383-92.

10. Nadal SR, Manzione CR. Manejo dos portadores das neoplasias intraepiteliais anais. Rev bras colo-proctol. 2008;28(4):462-4. https://doi.org/10.1590/50101-98802008000400009

11. Neto JCP, Silva TAA, Lago AFV, Rodrigues ABL, Nogueira NMCC, Bandeira WF, et al. Utilização do peeling químico nas alterações estéticas: uma revisão de literatura [Internet]. Teresina: Anais-Revista Interdisciplinar de Ciências Médicas; 2017. [citado 21 jan 2019]. Disponível em: https://gpicursos. com/interagin/gestor/uploads/trabalhos-feirahospitalarpia ui/4ab07057eb64e608cd0f2de5e12ba09c.pdf

12. Sociedade Brasileira de Dermatologia. Cauterização química [Internet]. Rio de Janeiro: SBD; 2016 [citado 19 jan 2019]. Disponível em: www.sbd.org.br/dermatologia/pele/ procedimentos/cauterizacao-quimica/2/

13. Zanini M. Gel de ácido tricloroacético - Uma nova técnica para um antigo ácido. Med Cutan Iber Lat Am. 2007;35(1):14-7.

14. Castro TMPPG, Duarte ML. Condiloma lingual: relato de caso clínico. Rev Bras Otorrinolaringol. 2004;70(4):565-8. https:// doi.org//10.1590/50034-72992004000400021

15. Conselho Regional de Enfermagem de São Paulo. Parecer Coren-SP 001/2015 de 07 de outubro de 2014 [Internet].
Brasília: 2015 [citado 21 jan 2019]. Disponível em: https:// portal.coren-sp.gov.br/wp-content/uploads/2015/04/ Parecer\%20001-2015.pdf

16. Ministério da Saúde (BR). Resolução n 466 de 12 de dezembro de 2012 [Internet]. Brasília, DF: 2012 [citado 18 abr 2017]. Disponível em: https://conselho.saude.gov.br/ resolucoes/2012/Reso466.pdf

17. Fernandes RM, Miguir ELB, Donoso TV. Perfil da clientela estomizada residente no município de Ponte Nova, Minas Gerais. Rev bras colo-proctol. 2010;30(4):385-92. https:// doi.org/10.1590/50101-98802010000400001

18. Macedo MS, Nogueira LT, Luz MHBA. Perfil dos estomizados atendidos em hospital de referência em Teresina. ESTIMA, Braz J Enterostomal Ther. 2005;4(4)25-8.

19. Boccardo LM, Nogueira AS, dos Santos ER, Miyadahira AMK, Santos VLCG. Aspectos da reinserção social do ostomizado. Rev esc enferm USP. 1995;29(1):59-71. https:// doi.org/10.1590/0080-6234199502900100059

20. Crema E, Silva R. Estomas: uma abordagem interdisciplinar. Uberaba: Pinti; 1997.

21. Santos Junior JCM. Câncer ano-reto-cólico: aspectos atuais II - Câncer colorretal - Fatores de riscos e prevenção. Rev bras colo-proctol. 2007;27(4):459-73. https://doi.org/10.1590/ S0101-98802007000400016

22. Instituto Nacional de Câncer. Estimativa 2018. Incidência de câncer no Brasil [Internet]. Rio de Janeiro: INCA; 2018 [citado 21 Jan 2019]. Disponível em: https://www.inca. gov.br/publicacoes/livros/estimativa-2018-incidencia-decancer-no-brasil

23. Mancini MC, Fiúza PM, Rebelo JM, Magalhães LC, Coelho ZAC, Paixão ML, et al. Comparação do desempenho de atividades funcionais em crianças com desenvolvimento normal e crianças com paralisia cerebral. Arq NeuroPsiquiatr. 2002;60(2-B):446-52. https://doi.org/10.1590/ S0004-282X2002000300020

24. Vilarinho RSC, Rogenski NMB, Rogenski KE. Gastrostomia: como cuidar. In: Cesaretti IUR, Paula MAB, Paula PR. Estomaterapia: temas básicos em estomas. São Paulo: Cabral; 2006. p. 243-50. 